\title{
Avaliação do Índice de Danos Permanentes através do SLICC/ACR-DI em Pacientes Brasileiros com Lúpus Eritematoso Sistêmico(*)
}

\section{Cumulative Organ Damage Evaluation Using the SLICC/ACR-DI in Brazilian Patients with Systemic Lupus Erythematosus}

\author{
Juliana Guarize ${ }^{(1)}$, Simone Appenzeller ${ }^{(2)}$ e Lilian Tereza Lavras Costallat ${ }^{(3)}$
}

\section{RESUMO}

Objetivo: avaliar a freqüência e os tipos de danos permanentes com relação ao tempo da doença em pacientes brasileiros com lúpus eritematoso sistêmico (LES) de um hospital universitário, através da aplicação do índice de dano do Systemic Lupus International Collaborating Clinics/American College of Rheumatology (SLICC/ ACR/DI). Métodos: foram incluídos 60 pacientes neste estudo. Os dados utilizados, extraídos dos prontuários dos pacientes, foram obtidos através de anamnese, exame fisico e exames complementares. A freqüência e os tipos de danos permanentes foram avaliados através do SLICC/ACR/DI. A estatística foi realizada através do teste quiquadrado e teste t. Regressão multivariada foi utilizada para correlacionar o dano com o tempo de doença. Resultados: entre os 60 pacientes, 41 (68,3\%) apresentaram algum tipo de dano. Houve tendência, entre os pacientes não caucasóides e com maior tempo de doença, a apresentarem mais danos $(\mathrm{p}=0,058)$. Os sistemas mais acometidos foram: pele (35,0\%); neuropsiquiátrica $(18,3 \%)$; ocular (15,0\%); vascularização periférica (16,6\%); e cardiovascular $(10,0 \%)$. Com o aumento do tempo de doença (superior a 60 meses), houve maior tendência a danos oculares, neuropsiquiátricos, renais, musculoesquelético e da pele. Com evolução superior a 120 meses houve aumento dos danos pulmonares, cardiovasculares e aqueles relacionados com a vascularização periférica. Conclusões: observou-se $68,3 \%$ de dano permanente, havendo maior freqüência de danos relacionados com acometimentos neuropsiquiátricos, oculares, da vascularização periférica e da pele. Não se observou importante acometimento renal e gonadal, ao contrário do descrito anteriormente. Pacientes não caucasóides apresentaram tendência a maiores escores de dano. No entanto, outros estudos são necessários para se comprovar o envolvimento de fatores étnicos e/ou fatores sócioeconômicos nesse processo de dano irreversível.

Palavras-chave: lúpus eritematoso sistêmico, dano, tempo de doença.

\begin{abstract}
Objective: to evaluate the frequency and the different types of organ damage in relation to disease duration in Brazilian patients with SLE followed in a tertiary reference center through the application of the SLICC/ACR-DI. Methods: sixty SLE patients were enrolled in this study, and relevant data were obtained through medical history, physical and laboratory examinations reviewing the hospital records. The frequency and different types of organ damage were determined using the SLICC/ACR-DI. Statistics were performed through qui-square and t-tests. Multivariate regression was used to correlate damage with disease duration. Results: forty-one patients (68.3\%) presented some cumulative organ damage. Non-caucasoid patients and patients with longer disease duration had a slight tendency to have more damage $(p=0.058)$. Skin (35.0\%), neuropsychiatric (18.3\%), ocular $(15.0 \%)$, peripheral vascularization $(16.6 \%)$ and cardiovascular (10.0\%) systems were more frequently affected. Patients with more than 60 months of disease had a slight tendency to present greater ocular, neuropsychiatric, renal, skin and musculoskeletal damage than patients with shorter disease duration. Patients with more than 120 months of disease had greater pulmonary, cardiovascular and peripheral vascular damage. Conclusions: in this study, $68.3 \%$ of permanent damage was observed. Skin, neuropsychiatric, ocular, peripheral vascularization and cardiovascular systems were more frequently affected. Renal and gonadal involvement was not as frequent as previously described. Non-caucasoid patients had a tendency to present higher scores, but more studies are necessary to determinate if ethnic or economic factors are involved.
\end{abstract}

Keywords: systemic lupus erythematosus, cumulative organ damage, outcome, prognosis.

\footnotetext{
* Trabalho realizado com bolsa de iniciação científica da Fundação de Amparo à Pesquisa do Estado de São Paulo (Fapesp) e com os Fundos Remanescentes da Sociedade Brasileira de Reumatologia. Recebido em 24/02/2003. Aprovado, após revisão, em 11/03/2004.

1. Sextanista de medicina da Faculdade de Ciências Médicas da Universidade Estadual de Campinas (FCM/Unicamp), SP.

2. Pós-graduanda do departamento de clínica médica da FCM/Unicamp.

3. Professora titular da disciplina de reumatologia da FCM/Unicamp.

Endereço para correspondência: Simone Appenzeller. Departamento de clínica médica, Faculdade de Ciências Médicas, Unicamp, CEP 13081-970, Campinas, SP.
} 


\section{INTRODUÇÃO}

O lúpus eritematoso sistêmico (LES) foi tradicionalmente considerado uma doença crônica, potencialmente fatal e com alta taxa de mortalidade ${ }^{(1-3)}$. Com o aumento da sobrevida, a morbidade secundária à doença e as possíveis complicações da terapia passaram a despertar maior interesse ${ }^{(4)}$. Os danos permanentes no LES podem ser decorrentes da própria doença (como, por exemplo, nefrite evoluindo para insuficiência renal; pneumonite evoluindo para fibrose pulmonar) ou do tratamento empregado, pois uma parcela significativa de pacientes recebe corticosteróides durante meses, anos ou a vida toda ${ }^{(1-3)}$. Os efeitos dos corticosteróides nos ossos podem levar à osteoporose e osteonecrose; no sistema cardiovascular, à hipertensão arterial sistêmica e aterosclerose, sendo importantes causas de morbi-mortalidade no $\operatorname{LES}^{(4)}$. Outros imunossupressores, como a azatioprina e a ciclofosfamida, freqüentemente empregados no LES, estão associados ao aumento de infecções, infertilidade e ao aparecimento de neoplasias anos após o início de seu uso ${ }^{(4)}$. A cloroquina, freqüentemente utilizada para manifestações cutâneas, pode causar uma maculopatia por impregnação da droga na retina e, se não reconhecida a tempo, pode ser responsável por alterações debilitantes na visão, como visão anormal para cores e embaçamento visual ${ }^{(4)}$.

Com a maior sobrevida dos pacientes com LES, observou-se o aparecimento de co-morbidades como o diabetes melito e a hipertensão arterial ${ }^{(1,4)}$. Ambos podem ser induzidos por corticosteróides, pela obesidade, mas também apresentam associação genética importante. A presença destas co-morbidades também aumenta a freqüência de danos permanentes, especialmente os relacionados com os sistemas cardiovasculares ${ }^{(4)}$.

Para avaliar o impacto destes tipos de dano, acumulados durante a evolução do LES, desde o seu início, foi desenvolvido o índice de dano do Systemic Lupus International Collaborating Clinics/American College of Rheumatology $(\text { SLICC/ACR/DI })^{(5)}$. Este índice foi primeiramente aplicado em caucasianos, hispânicos, afro-americanos, asiáticos e afro-caribenhos. Entre os países latino-americanos, foi primeiramente aplicado em pacientes mexicanos ${ }^{(6)}$.

O objetivo deste trabalho foi avaliar a freqüência e os tipos de dano permanentes em pacientes lúpicos brasileiros seguidos em um hospital universitário. Avaliou-se também a relação entre os diferentes tipos de danos permanentes e o tempo de doença.

\section{PACIENTES E MÉTODOS}

Foram estudados 60 pacientes que preenchiam quatro ou mais critérios para $\mathrm{LES}^{(7)}$, acompanhados no ambulatório de reumatologia do HC-Unicamp. Os pacientes foram selecionados aleatoriamente, não tendo havido qualquer seleção prévia quanto às suas características demográficas, manifestações clínicas, laboratoriais ou tratamento, no período entre janeiro e dezembro de 2001.

Quanto à duração da doença, considerada a partir da data do diagnóstico de LES, os pacientes foram previamente selecionados e divididos em 3 grupos, cada grupo constando de 20 pacientes:

Grupo 1: tempo de evolução da doença inferior a 60 meses;

Grupo 2: tempo de evolução da doença entre 60 e 120 meses;

Grupo 3: tempo de evolução da doença superior a 120 meses.

As características clínicas e laboratoriais, bem como os dados demográficos destes pacientes, foram obtidas a partir de seus prontuários. As medicações mais freqüentemente utilizadas no tratamento da doença e consideradas neste trabalho foram corticosteróides (oral [CE] e endovenoso [PCE]); antimaláricos (DCQ); e citostáticos (Azatioprina [AZA]; Ciclofosfamida oral [CFM] e pulsos endovenosos [PCFM]).

\section{CARACTERIZAÇÃO E AVALIAÇÃO DO DANO}

A avaliação do dano foi realizada de acordo com o SLICC/ ACR/DI, que avalia 12 itens de danos irreversíveis ocorridos desde o diagnóstico da doença, independentemente de serem advindos do LES, da terapêutica utilizada no tratamento ou de co-morbidades. Os danos foram considerados quando presentes lesões irreversíveis há no mínimo seis meses, não relacionadas com inflamação ativa e que ocorreram após o diagnóstico do LES, conforme previamente estabelecido ${ }^{(5)}$.

Para cada lesão observada foi somado um ponto, sendo que lesões repetidas somaram dois pontos, e a mesma lesão não pôde ser somada mais de uma vez. O somatório de pontos definiu o escore obtido.

O diagnóstico destas lesões foi feito através de exames rotineiramente empregados no HC-Unicamp, de acordo com as queixas clínicas e os exame fisico destes pacientes. A avaliação dos distúrbios cognitivos foi realizada através do exame do estado mental cognitivo ${ }^{(8)}$ e de um teste de memória lógica como anteriormente descrito ${ }^{(9)}$. 


\section{ANÁLISE ESTATÍSTICA}

Os pontos do SLICC/ACR/DI foram analisados pela normalidade, e os pacientes foram classificados em portadores ou não de danos e suas características examinadas, usando-se o teste qui-quadrado e o teste $\mathrm{t}$ de Student. Foi considerado o valor de $95 \%$ de significância. Para verificar associação do dano com o tempo de doença foi elaborado um modelo de regressão de Cox multivariado.

\section{RESULTADOS}

\section{CARACTERÍSTICAS DEMOGRÁFICAS}

A maioria dos pacientes estudados foi do sexo feminino (96,7\%). A média de idade foi de 38,46 anos (desvio padrão [DP] de 12,5). Houve maior freqüência de pacientes caucasóides (75\%) com relação à não caucasóides (25\%). O tempo médio de doença foi de 80 meses $(\mathrm{DP}=23,2)$ e o tempo médio de acompanhamento de 75 meses $(\mathrm{DP}=25,8)$. Neste trabalho não se observou diferença significativa entre as variáveis demográficas e a ocorrência de dano permanente, no entanto, notou-se tendência a maior escore de dano entre os pacientes não caucasóides $(p=0,058)$, sendo a média do escore de dano entre os pacientes não caucasóides de 2,133 (DP=0,568) e entre os pacientes caucasóides de 1,467 $(\mathrm{DP}=0,292)$.

Não houve diferença estatística entre outras variáveis demográficas nos grupos estudados, divididos em função do tempo da doença.

\section{MANIFESTAÇÕES CLÍNICAS E MEDICAÇÃO UTILIZADA}

Com relação às doenças associadas, observou-se aumento significativo da prevalência de hipertensão arterial sistêmica $(p<0,05)$ nos pacientes do grupo 3 , comparativamente aos pacientes dos grupos 1 e 2 . Não se observou diferença com relação às demais doenças, incluindo diabetes melito, síndrome de Sjögren, hiper ou hipotireoidismo e manifestações neurológicas. Com relação às medicações utilizadas, observou-se maior utilização de ciclofosfamida e pulsos de ciclofosfamida nos pacientes do grupo $3(\mathrm{p}<0,05)$ (Tabela 1$)$. Apesar de não ser estatisticamente significante, o uso de difosfato de cloroquina foi mais empregado no grupo 2. Não houve associação estatística entre os tipos de dano (Tabela 2) com as medicações estudadas.

\section{CARACTERIZAÇÃO E AVALIAÇÃO DO DANO}

Num total de 60 pacientes estudados, 41 (68,3\%) apresentaram algum tipo de dano permanente. Observou-se a seguinte
TABELA 1

LÚPUS ERITEMATOSO SISTÊMICO (LES):

MEDICAÇÃO UTILIZADA NOS TRÊS GRUPOS

\begin{tabular}{|c|c|c|c|c|}
\hline Medicação & Grupo $1(\%)$ & Grupo $2(\%)$ & Grupo $3(\%)$ & p \\
\hline $\begin{array}{l}\text { Corticosteróide } \\
\text { oral }\end{array}$ & $20(100 \%)$ & $20(100 \%)$ & $20(100 \%)$ & NS \\
\hline $\begin{array}{l}\text { Difosfato de } \\
\text { cloroquina }\end{array}$ & $13(65 \%)$ & $19(95 \%)$ & $16(80 \%)$ & NS \\
\hline Azatioprina & $7(35 \%)$ & $11(55 \%)$ & $6(30 \%)$ & NS \\
\hline $\begin{array}{l}\text { Ciclofosfamida } \\
\text { oral }\end{array}$ & $0^{*}$ & $4(20 \%)$ & $7(35 \%)^{*}$ & $<0,05$ \\
\hline $\begin{array}{l}\text { Pulso de } \\
\text { metilpredinisolona }\end{array}$ & $2(10 \%)$ & 0 & $1(5 \%)$ & NS \\
\hline $\begin{array}{l}\text { Pulso de } \\
\text { ciclofosfamida }\end{array}$ & $0^{*}$ & $3(15 \%)$ & $6(30 \%)^{*}$ & $<0,05$ \\
\hline
\end{tabular}

Grupo 1: tempo de doença <60 meses; grupo 2: tempo de doença entre 60-120 meses; grupo 3: tempo de doença $>120$ meses; NS: não significativo; * $p<0,005$ quando comparados os grupos 1 e 3

TABELA 2

LÚPUS ERITEMATOSO SISTÊMICO (LES): FREQUÊNCIA E TIPO DE DANO PERMANENTE EM 60 PACIENTES

\begin{tabular}{lc}
\hline Tipo de dano permanente & Freqüência (\%) \\
\hline Pele & $21(35,0)$ \\
Renal & $14(23,3)$ \\
Neuropsiquiátrico & $11(18,3)$ \\
Vascular periférico & $10(16,6)$ \\
Ocular & $9(15,0)$ \\
Cardiovascular & $6(10,0)$ \\
Pulmonar & $5(8,3)$ \\
\hline Musculoesquelético & $5(8,3)$ \\
Gastrointestinal & $2(3,3)$ \\
Diabetes melito & $1(1,7)$ \\
Neoplasias & $1(1,7)$ \\
\hline Falência gonadal prematura & $1(1,7)$ \\
\hline
\end{tabular}

distribuição de freqüência por sistemas acometidos: ocular em 9 pacientes (15\%); neuropsiquiátrico em 11 (18,3\%); renal em 14 (23,3\%); pulmonar em 5 (8,3\%); cardiovascular em 6 (10\%); vascularização periférica em 10 (16,6\%); gastrointestinal em 2 (3,3\%); musculoesquelético em 5 (8,3\%); pele em 21 (35\%); insuficiência gonadal em 1 (1,7\%); diabetes melito em 1 (1,7\%); e neoplasia em 1 (1,7\%).

Entre os danos encontrados na pele, o acometimento com cicatriz extensa foi o mais freqüente, ocorrendo em 19 de 21 pacientes. Entre os danos neurológicos o mais prevalente 
foi a alteração cognitiva, encontrada em 10 de 11 pacientes; trombose venosa, ulceração e estase foram as mais prevalentes com relação ao dano na vascularização periférica, encontrada em 6 de 10 pacientes; entre os danos oculares, o mais freqüente foi alteração da retina, em 5 de 9 pacientes, seguido de catarata, em 4 de 9 pacientes (Tabela 2).

Quando a ocorrência de dano é analisada com relação ao tempo da doença (Figura 1), observa-se que nos pacientes com até 60 meses de doença houve $65 \%$ de dano, sendo os mais freqüentes aqueles referentes à pele, à vascularização periférica e ao envolvimento neuropsiquiátrico. Dos pacientes com tempo de doença entre 60 e 120 meses, 75\% tinham algum tipo de dano, principalmente relativos à pele, ao envolvimento neuropsiquiátrico, ao renal e ao ocular. Dos pacientes com mais de 120 meses de doença, 65\% tinham algum tipo de dano, destacando-se os danos da pele e dos sistemas cardiovascular, da vascularização periférica e pulmonar (Figura 1).
Nesta casuística observou-se que dois $(3,3 \%)$ pacientes morreram antes de dez anos de doença, os quais tinham escores do SLICC/ACR-DI diferentes de zero. As causas da morte foram falência renal e infecção, com respectivamente 10 e 65 meses de doença.

\section{DISCUSSÃO}

Nas últimas quatro décadas, com o aumento na expectativa de vida dos pacientes com LES, tornou-se necessário avaliar a ocorrência de danos irreversíveis nos diferentes órgãos e sistemas, independente da natureza dos danos ${ }^{(4,5)}$. O SLICC/ ACR/DI ${ }^{(5)}$ elaborou um instrumento, separando os conceitos de dano e atividade da doença. Este índice foi desenvolvido especificamente para danos ou lesões acumulados ao longo da evolução da doença, oriundos diretamente do próprio LES, de doenças concomitantes e do seu tratamento ${ }^{(5)}$. Até o momento, este é o primeiro estudo que

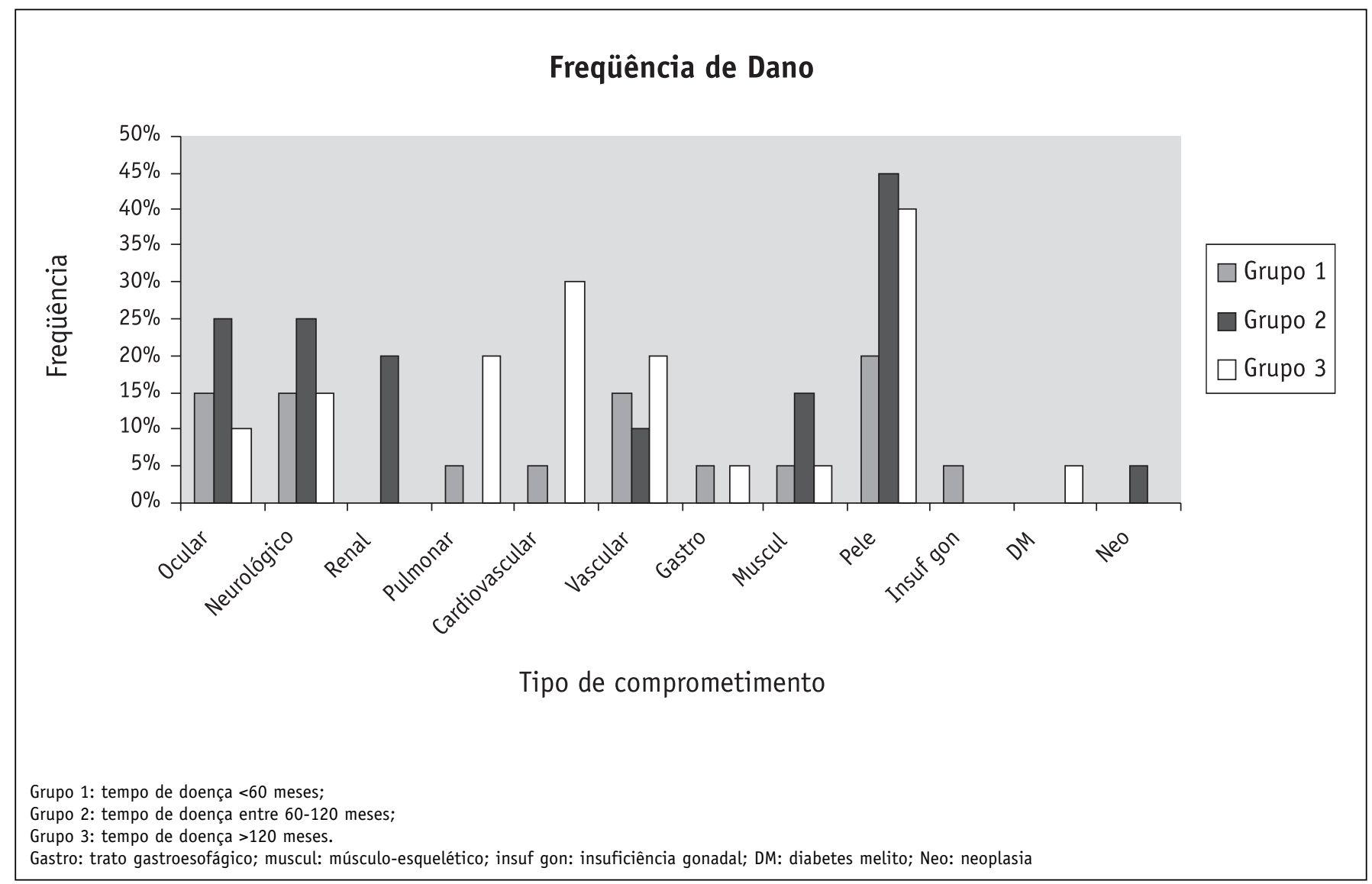

Figura 1 - Lúpus eritematoso sistêmico (LES): freqüência e tipos de danos com relação ao tempo da doença. 
utiliza o SLICC/ACR-DI em uma população brasileira, tendo sido observada elevada freqüência de danos permanentes, ocorrendo em $68,3 \%$ dos pacientes estudados. Os danos mais observados foram aqueles referentes à pele (35\%), ao envolvimento neuropsiquiátrico $(18,3 \%)$, ao ocular (15\%), à vascularização periférica $(16,6 \%)$ e ao cardiovascular (10\%).

Estudos $^{(6,10-14)}$ mostram que a freqüência de cada dano permanente está diretamente associada ao tempo da doença. Danos precoces, encontrados logo no início da doença, são causados diretamente pelo processo inflamatório do LES $^{(4,10)}$, enquanto as morbidades tardias sofrem um grande impacto da medicação empregada e de doenças concomitantes ${ }^{(4,11-14)}$. A mortalidade parece estar diretamente associada à ocorrência de dano, sendo que $25 \%$ dos pacientes com dano morrem antes de completar dez anos de doença ${ }^{(10)}$. Os fatores de risco associados à ocorrência de dano permanente incluem: idade avançada ao diagnóstico ${ }^{(6,10)}$; maior duração do $\mathrm{LES}^{(11)}$; etnia afro-caribenha ou asiática ${ }^{(15)}$; elevados índices de atividade da doença ao diagnóstico ${ }^{(6,10)} \mathrm{e}$ no decorrer da doença ${ }^{(12)}$.

Neste trabalho foi avaliado o dano com relação ao tempo da doença. Sobre os demais fatores de risco, não se observou diferença significativa neste grupo de pacientes, exceto com relação à raça. Embora não estatisticamente significativo, notou-se tendência a maiores valores de escore de dano permanente entre os pacientes não caucasóides, não sendo possível, contudo, estabelecer quais fatores étnicos estariam envolvidos nesta tendência ou também se há influência de fatores sócio-econômicos. Com o aumento do tempo da doença (superior a 60 meses), houve maior tendência a danos oculares, neuropsiquiátricos, renais, musculoesqueléticos e cutâneos. Nos pacientes com evolução superior a 120 meses houve aumento dos danos pulmonares, cardiovasculares e da vascularização periférica. Ao contrário do que foi relatado previamente ${ }^{(13)}$, observou-se envolvimento menor dos sistemas renal e gonadal nestes pacientes. Insuficiência gonadal prematura (antes dos 40 anos de idade) ocorreu numa prevalência de $5 \%$, sendo semelhante às casuísticas anteriores de pacientes não hispânicos ${ }^{(14)}$. A maior incidência de falência gonadal foi associada aos pulsos

\section{REFERÊNCIAS}

1. Cervera R, Khamashta MA, Font J, et al: Morbidity and mortality in systemic lupus erythematosus during a 10-year period: a comparison of early and late manifestations in a cohort of 1,000 patients. Medicine (Baltimore) 82:299-308, 2003. endovenosos de ciclofosfamida para tratamento de nefrite. O sistema neuropsiquiátrico foi o segundo mais afetado, como descrito anteriormente ${ }^{(5)}$, ocorrendo, principalmente, distúrbios cognitivos e neuropatia periférica. Nesta casuística observou-se o grande acometimento da pele, com cicatriz extensa, causado pelo processo inflamatório da doença ou secundário à infecção cutânea, como, por exemplo, herpes Zoster, não sendo relatada esta freqüência em trabalhos anteriores. A possibilidade de a maior exposição solar a que estão sujeitas as pacientes brasileiras (não obstante a recomendação de uso de fotoprotetores) potencializar o processo inflamatório é hipótese que pode ser levantada, mas não comprovada.

A terapia a longo prazo com prednisona aumenta o risco de morbidade e danos permanentes ${ }^{(13,14)}$. A dose acumulada elevada de prednisona está associada à fraturas por osteoporose, doença coronariana sintomática (angina) e catarata. Exposição a altas doses de prednisona por mais de dois meses subseqüentes associa-se à necrose avascular e à acidente vascular cerebral hemorrágico; pulsos de corticosteróides parecem estar associados à disfunção cognitiva ${ }^{(4,13,14)}$. Nesta casuística não foi encontrado, no entanto, fratura decorrente de osteoporose. Observou-se, porém, necrose avascular única e recorrente (ambas em articulação coxo-femural) em pacientes expostos à terapia com altas doses de prednisona (via oral) por mais de dois meses subseqüentes. Não houve associação entre medicações utilizadas e escores de dano mais elevados.

Em conclusão, nesta casuística de 60 pacientes brasileiros houve $68,3 \%$ de dano permanente, acometendo principalmente a pele (35\%), o sistema neuropsiquiátrico $(18,3 \%)$, ocular $(15 \%)$, vascularização periférica $(16,6 \%)$ e cardiovascular (10\%). Ao longo da doença, houve tendência a maior dano do sistema pulmonar, cardiovascular e vascularização periférica. Não se observou importante acometimento renal e gonadal, ao contrário do descrito anteriormente. Pacientes não caucasóides tiveram tendência a apresentar maiores escores de dano, no entanto, outros estudos são necessários para se comprovar a participação de fatores étnicos e/ou fatores sócio-econômicos nesse processo de dano irreversível.

2. Moss KE, Ioannou Y, Sultan SM, et al: Outcome of a cohort of 300 patients with systemic lupus erythematosus attending a dedicated clinic for over two decades. Ann Rheum Dis 61: 409-13, 2002.

3. Trager J, Ward MM: Mortality and causes of death in systemic lupus erythematosus. Curr Opin Rheumatol 13:345-51, 2001. 
4. Petri M: Hopkins Lupus Cohort. Rheum Dis Clin North Am 26: 199-213, 2000

5. Gladman D, Glinzler E, Goldsmith C, et al: The development and initial validation of the Systemic lupus International Collaborating Clinics/ American College of Rheumatology damage index for systemic lupus erythematosus. Arthritis Rheum 39: 363-78, 1996.

6. Zonana-Nacach A, Camargo-Coronel A, Yanez P, et al: Measurement of damage in 210 mexican patients with systemic lupus erythematosus: relationship with disease duration. Lupus 7: 119-23, 1998.

7. Tan EM, Cohen AS, Fries JF, et al: The 1982 revised criteria for the classification of systemic lupus erythematosus. Arthritis Rheum 25: 1271-7, 1982.

8. Folstein MF, Folstein SE, Folstein PR: "Mini mental state" A practical method for grading the cognitive state of patient for the clinician. J Psychiatr Res 12: 189-98, 1975.

9. Appenzeller S, Fujisawa G, Costallat LTL: Distúrbios cognitivos no lúpus eritematoso sistêmico. Rev Bras Reumatol 39: 12-8, 1999.
10. Rahman P, Glagman DD, Urowitz MB, et al: Early damage as measured by the SLICC/ACR damage index is a predictor of mortality in systemic lupus erythematosus. Lupus 10: 93-6, 2001.

11. Stoll T, Seifert B, Isenberg DA: SLICC/ACR damage index is valid, and renal and pulmonary organ scores are predictors of severe outcome in patients with SLE. Br J Rheumatol 35: 248-5, 1996.

12. Stoll T, Stucki G, Malik J, et al: Association of the SLICC/ACR damage index with measures of disease activity and health status in patients with SLE. J Rheumatol 24: 309-13, 1997.

13. Zonana-Nacach A, Barr SG, Magder LS, et al: Damage in systemic lupus erythematosus and its association with corticosteroids. Arthritis Rheum 43: 1801-8, 2000.

14. Gorgos L, Goldman D, Petri M: The ACR/SLICC damage index in SLE. Arthritis Rheum 36: S68, 1993.

15. Karlson EW, Daltroy LH, Lew RA, et al: The relationship of socioeconomic status, race, and modifiable risk factor to outcomes in patientes with SLE. Arthritis Rheum 40: 47-56, 1996. 